\title{
Variation of Radiation Emission with Argon Gas Pressure in UM Plasma Focus with the Hollow Anode
}

\author{
Muhammad Zubair Khan ${ }^{1,2}$, Yap Seong Ling ${ }^{1}$, Wong Chiow San ${ }^{1}$ \\ ${ }^{1}$ Plasma Technology Research Center, Department of Physics, Faculty of Science, \\ University Malaya, Kuala Lumpur, Malaysia \\ ${ }^{2}$ Department of Physics, Federal Urdu University of Arts, Science \& Technology, Islamabad Pakistan \\ Email: mzubairkhan_um76@yahoo.com
}

Received August 16, 2012; revised February 25, 2013; accepted March 5, 2013

Copyright (C) 2013 Muhammad Zubair Khan et al. This is an open access article distributed under the Creative Commons Attribution License, which permits unrestricted use, distribution, and reproduction in any medium, provided the original work is properly cited.

\begin{abstract}
A Plasma Focus device $(2.2 \mathrm{~kJ}, 12 \mathrm{kV})$ is studied as a pulsed X-ray source, operated with Argon at a filling pressure in the range of 0.7 to 2.5 mbar. The time resolved X-ray signals are measured with an array of PIN diode detectors. The $\mathrm{X}$-ray emission produced by the plasma focus discharge at various pressures is investigated and compared. It is found that at the high pressure regime of more than 1.5 mbar, very consistent and high output of X-ray radiation is obtained, at the peak of the discharge current. A remarkable increase of about five times of the average X-ray yield is achieved at optimum pressure 1.7 mbar compared to that obtained at other pressures. An indirect method to determine the electron temperature of the plasma is achieved by using the array of 5 channel PIN diode detector coupled with $\mathrm{Al}$ foil of different thicknesses. The result shows that the electron temperature of the plasma is $7 \mathrm{keV}$, when the operating pressure is at 1.7 mbar. The maximum total X-ray yield is about $2.53 \mathrm{~mJ}$ per shot at optimum pressure, equivalent to the efficiency of $0.00012 \%$.
\end{abstract}

Keywords: Plasma Focus; UM Plasma Focus; Radiation Emission; Electron Temperature; X-Ray Yield; Instabilities

\section{Introduction}

A short lived but high density and high temperature plasma is produced at the final phase of the plasma focus discharge, which is a copious source intense X-ray radiation. Typically, the plasma temperature is higher than 1 $\mathrm{keV}$ range and the radiation emitted is mainly due to bremsstrahlung emission for pinches of Hydrogen and its isotopes or Helium. For higher atomic number gases, the radiation emitted is dominated by line radiation. The electron temperature of the plasma focus can be indirectly determined by analyzing the spectrum of the radiations [1]. Bernstein [2] made the time resolved measurement of the emitted spectra of the deuterium plasma focus discharge to find the correlation of the X-ray and the neutron emission. They found that several periods of $\mathrm{X}$-ray of different energy were possibly corresponding to the different period of the neutron emission. Two period of neutron emission are typically observed in the plasma focus discharge, they are associated with the thermal and acceleration mechanism [3]. A report of the measurement of the soft X-ray emission was shown several period of weak X-ray emission from low energy plasma focus of
$345 \mathrm{~J}$ with Hydrogen of 7 mbar of pressure. The soft $\mathrm{X}$-ray pulses were generally correlated in time with the peak voltage and current dip, which indicated the pinch phase and post-pinch phase [4]. Sh Al-Hawat et al. [5] studied the influence of copper impurities on the determination of electron temperature of the low energy Mather type plasma $(2.8 \mathrm{~kJ}, 15 \mathrm{kV})$ in AECS PF-2. They used the X-ray ratio method with different $\mathrm{Al}$ foil thicknesses to determine the electron temperature with Argon working gas. Five channel BPX 65 PIN diode was employed to record the X-ray signals. They compared the ratio of experimentally obtained shot values with the ratio of theoretically calculated values for Argon plasma. $\mathrm{X}$-ray emission ratio was $\mathrm{Cu}-\mathrm{K} \alpha$ radiation for the most of discharges, and determined the electron temperature of $1.5-2.5 \mathrm{keV}$ for the Argon plasma. Khan et al. [6] studied the radiation emission in low energy $(2.2 \mathrm{~kJ}, 12 \mathrm{kV})$ plasma focus, where they introduced the pragmatic consequence of ionized gas particles so-called plasma interference profile impact on the radiation emission which is new parameter that has significant effect on the yield of radiation in the dense plasma focus devices. Hussain et al. [7] measured the X-ray yield in different energy range as 
function of filling pressure of Hydrogen gas and found maximum X-ray yield $46.6 \mathrm{~J}$ with wall plug efficiency $1.40 \%$ in $4 \pi$ geometry. They presented a fine structure specimen of X-ray radiogram of a fish regarding high intensity $\mathrm{X}$-ray source for contrast biological radiography.

In our present paper, we report the higher X-ray yield while operating our plasma focus device at higher pressure of working gas Argon, where 1.7 mbar is optimized pressure. The array of the PIN diode detector is used to measure the time resolved X-ray signals and to estimate the corresponding electron temperature of the plasma by using the ratio. The high temperature obtained also suggests the presence of energetic electron beam.

\section{Experimental Complex}

The experiment procedures were carried out on the Mather-type Plasma Focus device located at the University of Malaya. The PF device is energized by a $30 \mu \mathrm{F}$ Maxwell capacitor charged up to $12 \mathrm{kV}$. The measured total external inductance is $165 \mathrm{nH}$. The schematic of $2.2 \mathrm{~kJ}$ Plasma Focus device and the five channel BPX65 PIN diode spectrometer are shown in Figure 1. The discharge tube consists of an inner electrode, made of hollow copper cylinder with diameter of $1.9 \mathrm{~cm}$, and length of 18 $\mathrm{cm}$. The hollow anode is used because the studies of energy of electron beam in the plasma focus device. The outer electrode is composed of six copper rods which form the shape of a squirrel cage with an inner diameter of $3.2 \mathrm{~cm}$ and the photo of the system is presented in Figure 2. The length of the individual cathode rod is 27 $\mathrm{cm}$. The cathode rod length is $9 \mathrm{~cm}$ higher than the length of anode rode. Hollow Anode and cathode are separated by a Pyrex glass insulator of $5 \mathrm{~cm}$ length. The vacuum system consists of a rotary van pump which evacuates the chamber to lower than $10^{-2}$ mbar before puffing Argon gas. As the gas contamination with impurities have a significant effect on output radiation, the chamber is refreshed after every 5 shots and fresh Argon gas is refilled to the desired pressure.

Identical coaxial cables of length $110 \mathrm{~cm}$ were used for all electrical diagnostics. All the coaxial cables were shielded with aluminum foilin order to reduce the effects of electromagnetic (EM) noise on data signals. Two DPO 4043 digital storage oscilloscope were used to record the

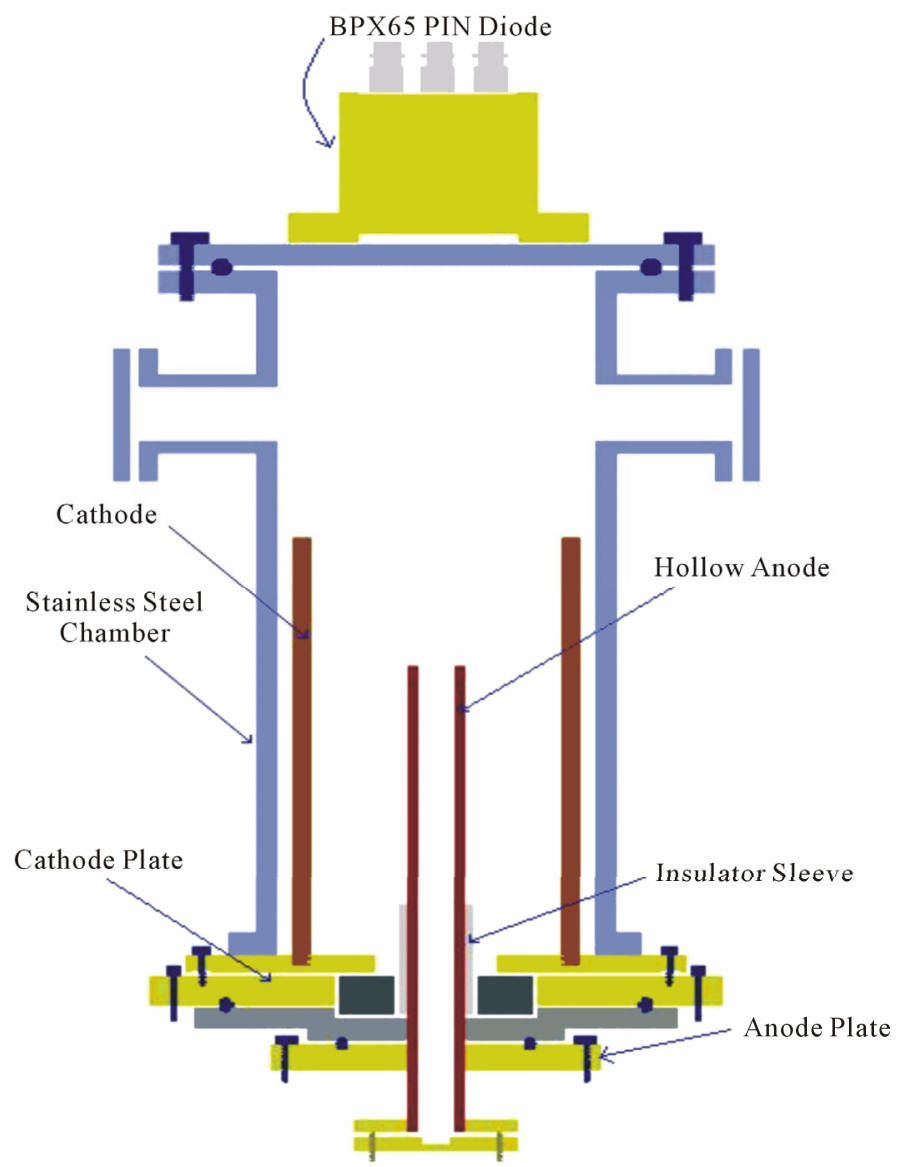

(a)

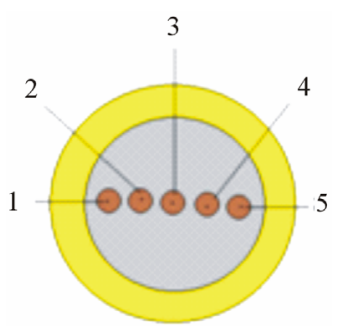

(b)

Figure 1. Schematic of UM-PF device (a); and five channels BPX65 PIN diode (b). 


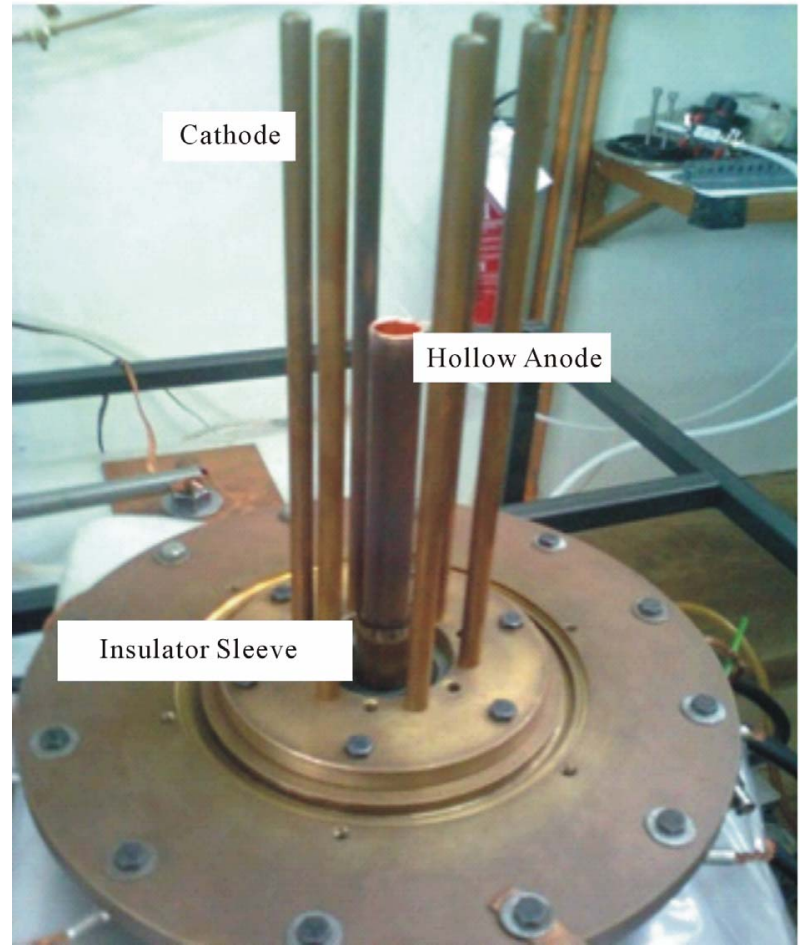

(a)

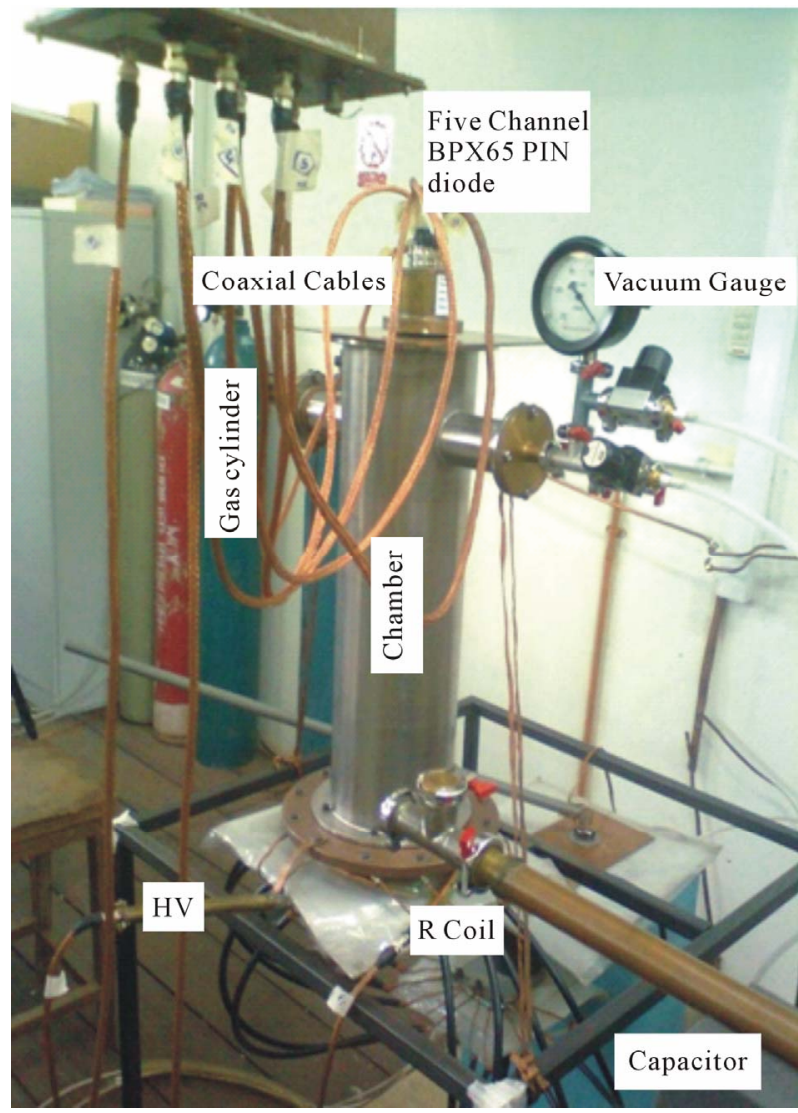

(b)

Figure 2. Inner hollow anode and outer cathode arrangement (a); and UM-PF system (b). total electrical signal from the Rogowski coil, high voltage probe and the five channel PIN diode's signals. Oscilloscope was triggered simultaneously for all signals. The five channel PIN diode detector was normalized against each other by masking each with an identical foil of Aluminized Mylar of $23 \mu \mathrm{m}$ thickness. The PIN diode response is between 1 and $30 \mathrm{keV}$. Combination of filters was used to look at different spectral windows.

\section{Results and Discussion}

The X-ray emission from the plasma focus operated with Argon is investigated by time resolved PIN diode detector (five channel BPX65 PIN diode). System design parameters are summarized in Table $\mathbf{1}$ at $12 \mathrm{kV}$ of UM-PF system.

The total discharge current and voltage was measured by a Rogowski coil and high voltage probe respectively. The typical signal of Rogowski coil and high voltage probe is shown in Figure 3.

For the detection of X-rays, an array of five filtered PIN diode detector (BPX-65) was housed at $43.50 \mathrm{~cm}$ far from the hollow anode head to measure the radiation emission from focused plasma. For X-ray detection the glass window of the PIN diode was detached. The PIN diode response is between 0.5 and $30 \mathrm{keV}$ [8]. These detectors were normalized against each other by masking all of them with different thickness of filters. The window of each diode was covered with Al foil and those thicknesses of each of them were given in Table 2.

The bias circuit of the BPX65 diode is depicted in Figure 4 and can be seen the PIN diodes are reverse biased at -45 volts.

The transmission curves of the BPX-65 PIN diode

Table 1. Design parameters of UM-PF system.

\begin{tabular}{cccc}
\hline Components & Length $(\mathrm{cm})$ & Diameter $(\mathrm{cm})$ & Material \\
\hline Hollow anode & 18.00 & $1.90 / 1.60($ O.D/I.D) & Copper \\
Cathode rod & 27.20 & 0.95 & Copper \\
Insulator sleeve & 5.00 & 2.00 & Pyrex \\
\hline
\end{tabular}

Table 2. An array of five PIN photodiodes exposed with Al + Aluminized Mylar $(\mu \mathrm{m})$.

\begin{tabular}{ccc}
\hline PIN diode & Filter & Thickness $(\mu \mathrm{m})$ \\
\hline 1 & Aluminized Mylar & 23 \\
2 & Al + Aluminized Mylar & $23+20$ \\
3 & Al + Aluminized Mylar & $23+30$ \\
4 & Al + Aluminized Mylar & $23+40$ \\
5 & Al + Aluminized Mylar & $23+100$ \\
\hline
\end{tabular}




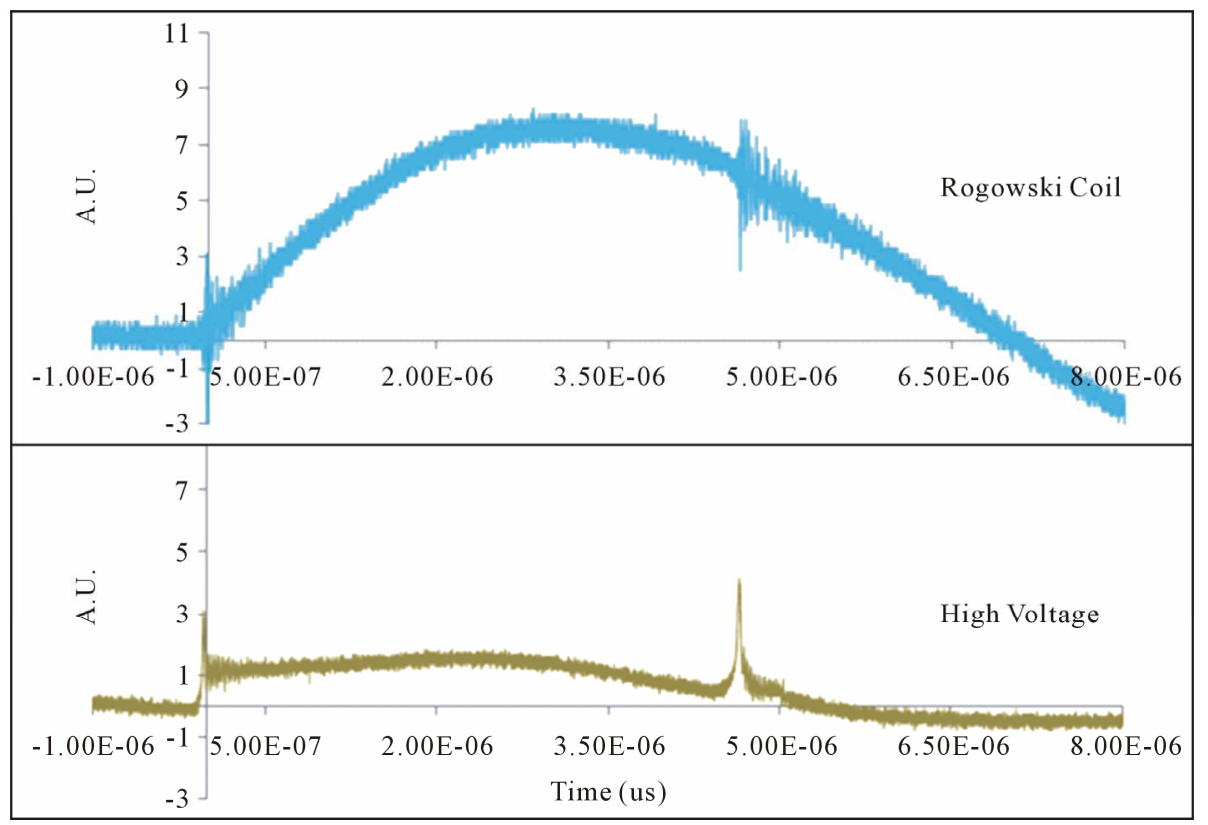

Figure 3. The typical signal of Rogowski coil and high voltage probe.

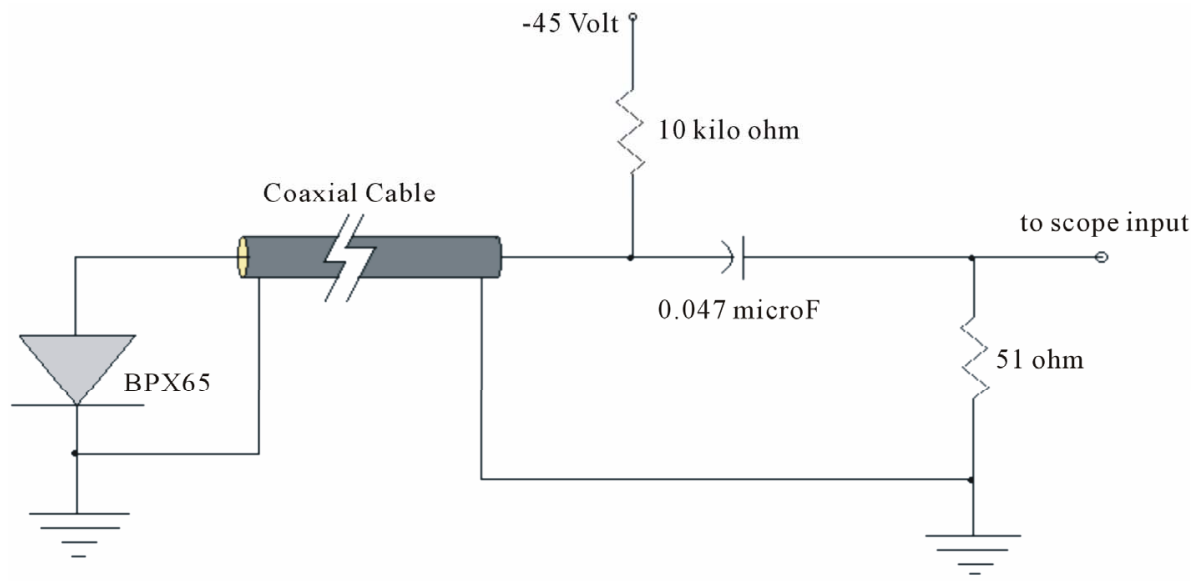

Figure 4. The basic circuit of the BPX65 PIN photodiode.

along with their associated absorption filters are illustrated in Figure 5.

The five channel PIN diode X-ray spectrometer masked with different thicknesses of $\mathrm{Al}$ foil may help to estimate the X-ray yield in $4 \pi$-geometry, and the system efficiency for X-ray generation. Energy radiated as X-ray is determined by formula [9].

$$
Y=\frac{Q_{\exp }(4 \pi)}{\mathrm{d} \Omega S(E) T(E)}
$$

where, $Q_{\exp }=\int \frac{V \mathrm{~d} t}{R}(\mathrm{C}),\left(\int V \mathrm{~d} t\right.$ is the area under the signals with five channel BPX 65 PIN diode's filters and $R$ is the $50 \Omega$ (in our experiments)), $S(E)$ is the average sensitivity of the detector, $T(E)$ is the average transmis- sion of the filter; and $\mathrm{d} \Omega=\mathrm{d} A / r_{o}^{2} \quad$ (sr.) is the solid angle subtended by the detector at the center of the anode where $\mathrm{d} A=\pi r^{2}$, in which $r(\mathrm{~cm})$ is the radius of the exposed area of each detector while $r_{o}(43.50 \mathrm{~cm})$ is the distance from the detector to the hollow anode.

The variation of X-ray emission can play effective role in generation of radiation in UM-PF deviceas a function of Argon filling pressure. Within our experiment, for instance, a pair of Ross filter $(20 \mu \mathrm{m} \mathrm{Al}$ foil, $100 \mu \mathrm{m} \mathrm{Al}$ foil) is used to determine the total radiation (X-ray) yield. The variation of average signal intensity with the 1.7 mbar pressure of Argon filling gas is described in Figure 6. It is found that the average signal intensity recorded by this pair of Ross filter attains its maximum value at 1.7 mbar Argon gas pressure.

Figure 7 shows the variation of total X-ray yield and 


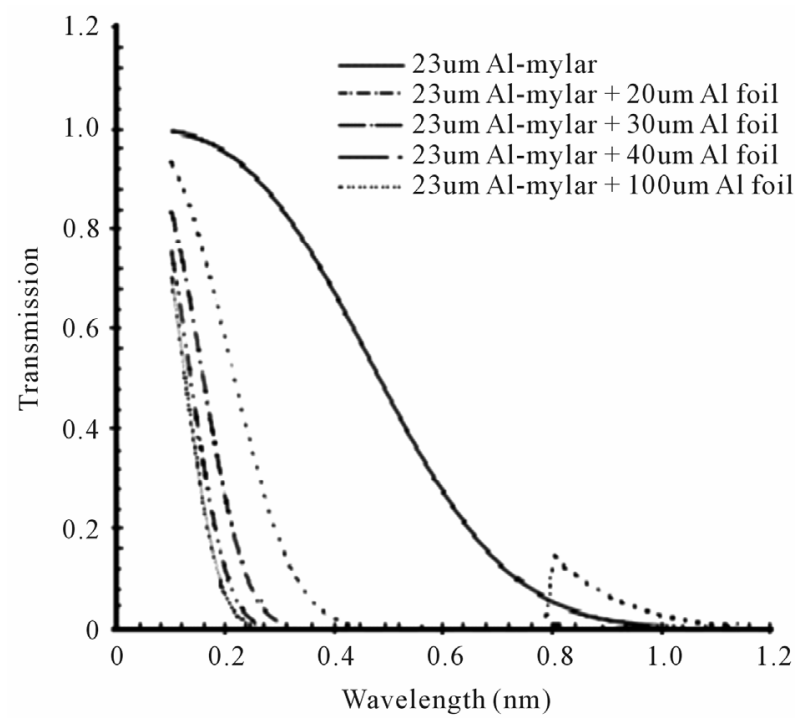

Figure 5. Transmission curves of $23 \mu \mathrm{m}$ aluminized mylar, $23 \mu \mathrm{M}$ aluminized mylar $+(20 \mu \mathrm{m}, 30 \mu \mathrm{m}, 40 \mu \mathrm{m}$, and 100 $\mu \mathrm{m})$ Al foil.

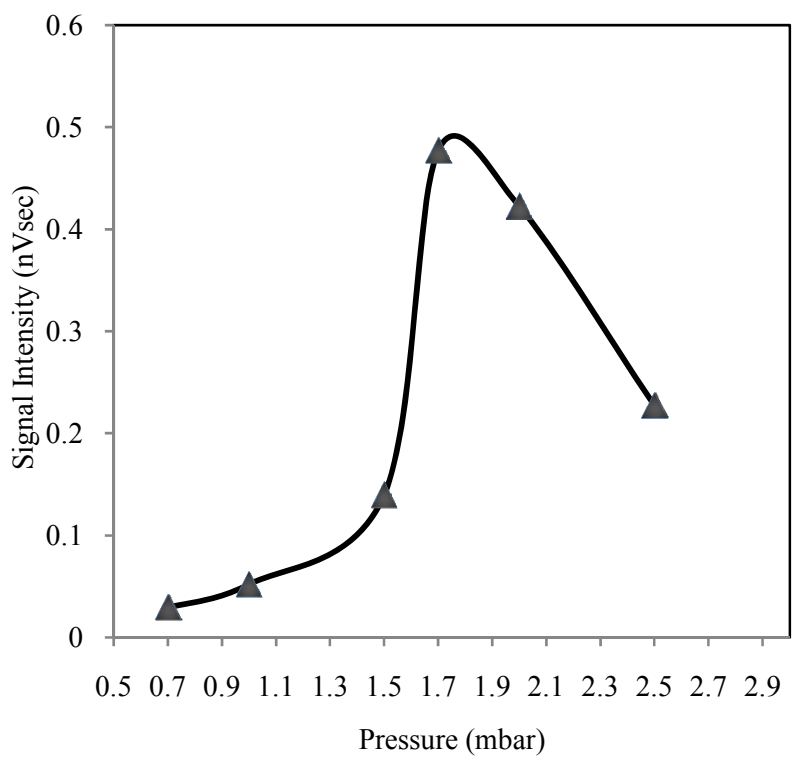

Figure 6. Variation of signal intensity recorded by Al foil versus Argon gas pressure.

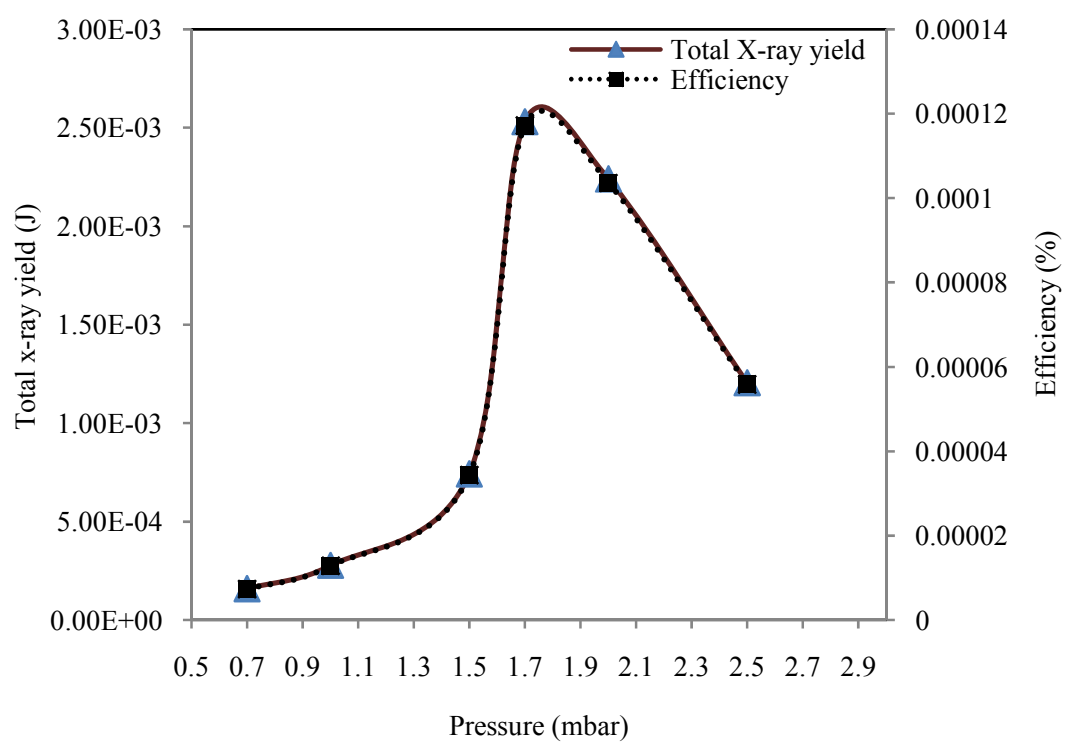

Figure 7. Variation of total X-ray yield in $4 \pi$-geometry with efficiency versus Argon gas pressure at constant voltage $12 \mathrm{kV}$.

efficiency against the Argon gas pressure with constant voltage $12 \mathrm{kV}$. Maximum X-ray yield in $4 \pi$-geometry is $2.53 \mathrm{~mJ}$ with corresponding efficiency of $0.00012 \%$ for optimized pressure 1.7 mbar with constant voltage $12 \mathrm{kV}$. After a series of experiments, we got optimized pressure 1.7 mbar at constant voltage $12 \mathrm{kV}$ by using a pair of thickness of Aluminium foil $(20 \mu \mathrm{m} \mathrm{Al}$ foil, $100 \mu \mathrm{m} \mathrm{Al}$ foil). Our Al foils, which are exactly on the top of hollow anode tip at distance $43.50 \mathrm{~cm}$. In case of $18 \mathrm{~cm}$ long hollow anode, electron beam from the focus region will interact with surface of anode, so radiation emit from focus and after hitting with the surface of anode is pass through the Aluminium foils thickness. The total X-ray yield is $2.53 \mathrm{~mJ}$ from this pair of $\mathrm{Al}$ foil.

It is very motivating outcome that the maximum electron temperature $7 \mathrm{keV}$ and the maximum radiation (Xray) yield $2.53 \mathrm{~mJ}$ is found at the same optimized Argon gas filling pressure 1.7 mbar of our UM-PF device. Our Plasma focus device has hollow anode as shown is Figure 2, thus radiation emission is less as compared to the solid anode because of less interaction on the surface of anode.

The ratio $R=I / I_{0}$ (where $I$ and $I_{0}$ are intensities) against different Al foil thicknesses (in Table 2) for the range of electron temperature from $3 \mathrm{keV}$ up to $7 \mathrm{keV}$ with pressure range 1.0 mbar to 2.5 mbar. Here, in Fig- 
ure 8, the estimated electron temperature from ratio method is found around $3 \mathrm{keV}$, when the Argon gas pressure is 1.5 mbar. In Figure 9, the electron temperature is $7 \mathrm{keV}$ or around $8 \mathrm{keV}$ with the Argon gas pressure 1.7 mbar. Figure 10 shows the electron temperature $6 \mathrm{keV}$ with the Argon gas pressure 2.0 mbar.

The existence of the Rayleigh-Taylor (RT) instability in the compression period of the plasma focusis established with the aid of the interferometric studies [10]. The interface between the plasma and the magnetic field is found to be unstable during radial compression, where the inertial force inside the plasma column is in the direction normal to the magnetic field. The electron and ions to drift in opposite direction is due to the charge independent force interacts with the magnetic field. A charge separation in the surface layer of the plasma column is due to this drift and is responsible to establish an E-field, which then introduces a further plasma drift. Therefore, the system becomes unsteady and this moment is referred to as the commencement of RT instability. The boundary of plasma column is fluted due to RT instability at the end of the compression phase. The growth time of the RT instability is about $30 \mathrm{~ns}$ [11].

It has been extensively evidenced in plasma focus device for a particular gas at its optimum pressure since the instability accelerated electron beam be more energetic. Thus the electron temperature is expected to be highest at optimum pressure. At higher than optimum pressure even though the plasma density is high, a consequence is observed which tends to suppress the $\mathrm{m}=0$ sausage instability. As an effect of dampening in the RT instability creation, the electron temperature is expected to be low because a substantial factor of the pinch current begins to flow through the rarefied pinch plasma. In our experimental finding, the measured higher electron temperature is $7 \mathrm{keV}$ at the optimum pressure 1.7 mbar of working gas Argon, which corresponds above theory.

Within the experiments, we use a constant voltage 12 $\mathrm{kV}$ for the determination of electron temperature. The electron temperature using different Argon gas pressure (1.5 mbar, 1.7 mbar, and 2.0 mbar) is found to vary in the range of $3 \mathrm{keV}$ to $7 \mathrm{keV}$. The highest electron temperature and the highest total X-ray yield is $7 \mathrm{keV}$ and 2.53 $\mathrm{mJ}$ at 1.7 mbar Argon gas pressure, respectively. It is very remarkable result using hollow copper anode in UM plasma focus device.

\section{Conclusion}

The present study confirms the significance of Argon filling gas pressure on the radiation emission in a plasma focus. For plasma focus device, the electron temperature exponentially decays with increase in pressure after optimized pressure, which is responsible to set up the instabilities, as RT instability plays significance role at higher pressure than the optimized pressure 1.7 mbar of Argon

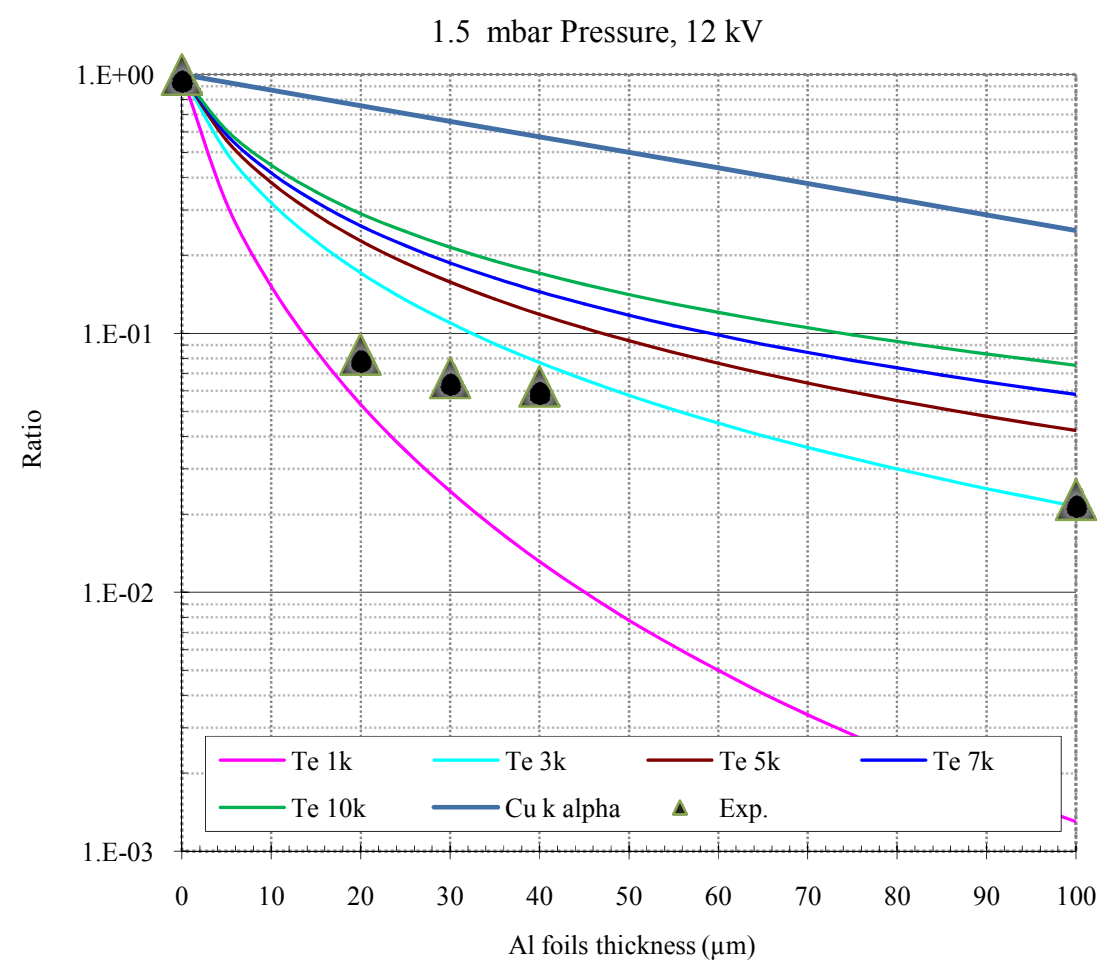

Figure 8. Calculated absorption curves of Al foils for $\mathrm{X}$-rays from copper plasma at various temperature and $\mathrm{Cu}$-K $\alpha \mathrm{X}$-rays with Argon gas pressure 1.5 mbar, estimated electron plasma temperature $3 \mathrm{keV}$. 


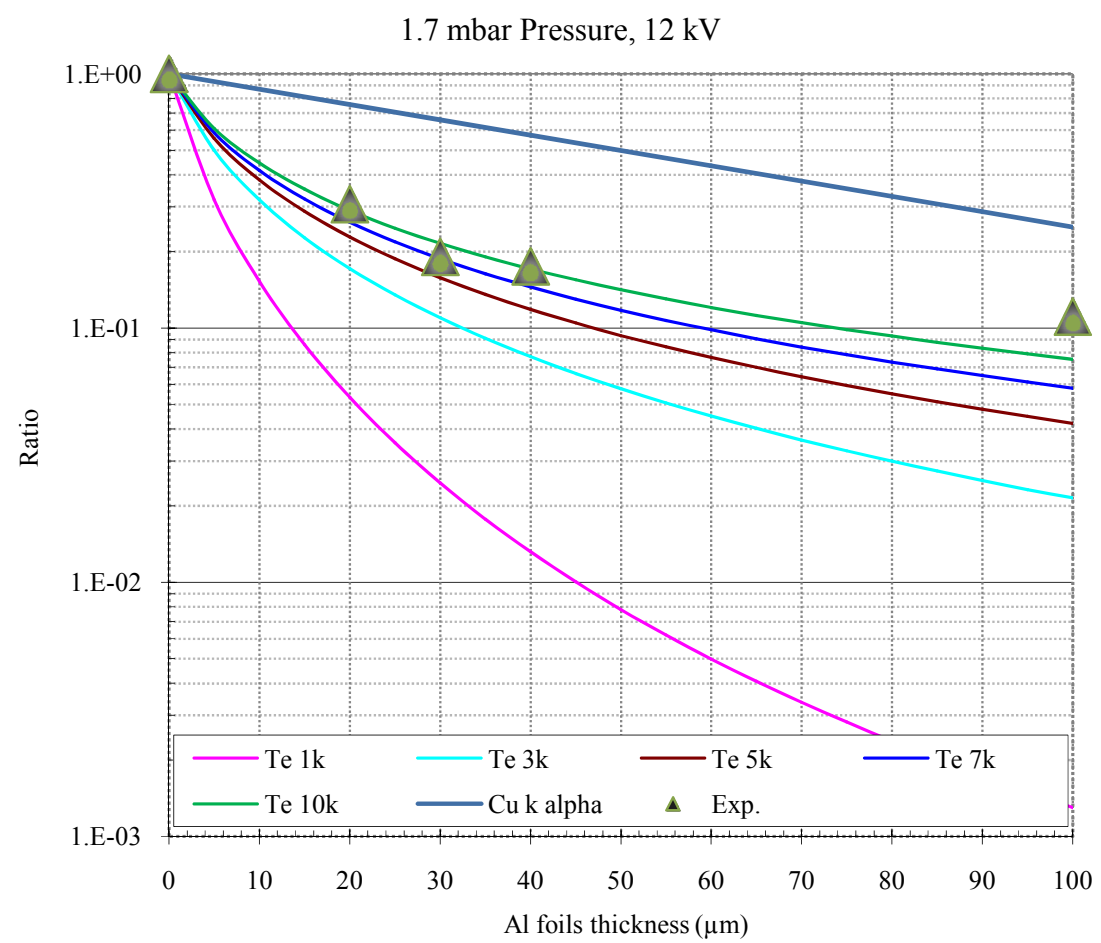

Figure 9. Calculated absorption curves of Al foils for $\mathrm{X}$-rays from copper plasma at various temperature and $\mathrm{Cu}$-K $\alpha \mathrm{X}$-rays with Argon gas pressure 1.7 mbar, estimated electron plasma temperature $7 \mathrm{keV}$.

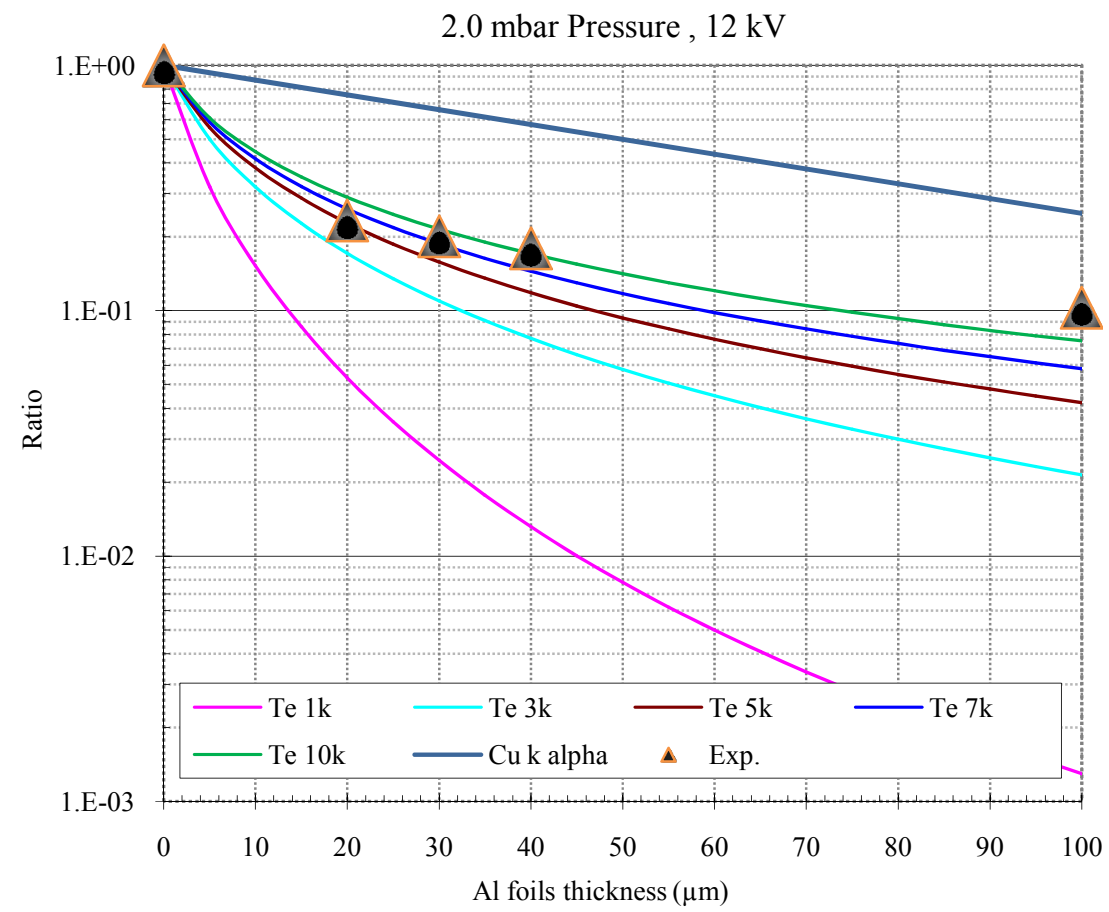

Figure 10. Calculated absorption curves of Al foils for X-rays from copper plasma at various temperature and $\mathrm{Cu} K \boldsymbol{K} \mathrm{X}$-rays with Argon gas pressure $2.0 \mathrm{mbar}$, estimated electron plasma temperature $6 \mathrm{keV}$.

gas. Our estimated highest electron temperature is $7 \mathrm{keV}$ and maximum total X-ray yield is $2.53 \mathrm{~mJ}$ with efficiency $0.00012 \%$, at 1.7 mbar optimized Argon pressure, in a low energy plasma focus $(2.2 \mathrm{~kJ}, 12 \mathrm{kV})$. The char- acteristic of Aluminum foil is found to be a good choice to diagnose hot plasma. The findings are extremely remarkable using hollow copper anode with novel dimension in our plasma focus device. The results will be use- 
ful for the academic studies as well as for future application of the electron beam emission from the plasma focus device.

\section{Acknowledgements}

Authors wish to acknowledge Mr. Lim and Mr. Jasbir for persist in during the experiment and technical support. Authors also wish to acknowledge the excellent support set by Federal Urdu University of Arts, Science \& Technology (FUUAST) Islamabad Pakistan for continuing smooth study in University of Malaya (UM) Kuala Lumpur Malaysia.

\section{REFERENCES}

[1] F. C. Jahoda, E. M. Little, W. E. Quinn, G. A. Sawyer and T. F. Stratton, "Continuum Radiation in the X-ray and Visible Regions from a Magnetically Compressed Plasma (Scylla)," Physical Review, Vol. 119, No. 3, 1960, pp. 843-856. doi:10.1103/PhysRev.119.843

[2] M. J. Bernstein, "Acceleration Mechanism for Neutron Production in Plasma Focus and z-Pinch Discharges," Physics of Fluids, Vol. 13, No. 11, 1970, pp. 2858-2866. doi:10.1063/1.1692871

[3] S. L. Yap, C. S. Wong, P. Choil, C. Dumitrescul and S. P. Moo, "Observation of Two Phases of Neutron Emission in a Low Energy Plasma Focus," Japanese Journal of Applied Physics, Vol. 44, No. 11, 2005, pp. 8125-8132. doi:10.1143/JJAP.44.8125

[4] K. Ranjini, P. Y. Nabhiraj, S. K. Das, C. Mallik and R. K. Bhandari, "Estimation of Electron Temperature in 14.45 GHz ECR Ion Source Plasma by Analysis of Bremsstrahlung Spectra," Indian Journal of Pure \& Applied
Physics, Vol. 45, No. 12, 2007, pp. 965-968.

[5] Sh. Al-Hawat, M. Akel and C. Wong, "X-Ray Emission from Argon Plasma Focus Contaminated with Copper Impurities in AECS PF-2 Using Five Channel Diode Spectrometer," Journal of Fusion Energy, Vol. 30, No. 6, 2011, pp. 503-508.

[6] M. Z. Khan, S. L. Yap, and C. S. Wong, "The Impact of Plasma Interference Profile (PIP) on Argon Discharge in Plasma Focus Device," International Journal of Physical Sciences, Vol. 8, No. 8, 2013, pp. 286-294.

[7] S. Hussain, S. Ahmad, M. Z. Khan, M. Zakaullah and A. Waheed, "Plasma Focus as a High Intensity Flash X-Ray Source for Biological Radiography," Journal of Fusion Energy, Vol. 22, No. 3, 2003, pp. 195-200. doi:10.1023/B:JOFE.0000037787.36243.b1

[8] M. Zakaullah, K. Alamgir, M. Shafiq, M. Sharif, A. Waheed and G. Murtaza, "Low-Energy Plasma Focus as a Tailored X-Ray Source," Journal of Fusion Energy, Vol. 19, No. 2, 2000, pp. 143-157. doi:10.1023/A:1013970229271

[9] M. Zakaullah, K. Alamgir, G. Murtaza and A. Waheed, "Efficiency of Plasma Focus for Argon K-Series Line Radiation Emission," Plasma Sources Science \& Technology, Vol. 9, No. 4, 2000, pp. 592-596. doi:10.1088/0963-0252/9/4/315

[10] N. J. Peacock, M. G. Hobby and P. D. Morgan, "Measurements of the Plasma Confinement and Ion Energy in the Dense Plasma Focus," 4th IAEA Conference on Plasma Physics and Controlled Nuclear Fusion Research, Madison, 17-23 June 1971, pp. 537-551.

[11] A. Jeffery and T. Taniuti, "Mhd Stability," Magnetohydrodynamic Stability and Thermonuclear Confinement, Academic Press, New York, 1996, pp. 304-305. 